Article

\title{
Reduced n-3 and n-6 PUFA (DHA and AA) Concentrations in Breast Milk and Erythrocytes Phospholipids during Pregnancy and Lactation in Women with Obesity
}

\author{
Rodrigo Chamorro $^{1}\left(\mathbb{D}\right.$, Karla A. Bascuñán ${ }^{1} \mathbb{D}$, Cynthia Barrera ${ }^{1}$, Jorge Sandoval ${ }^{2}$, Claudia Puigrredon $^{2}$ \\ and Rodrigo Valenzuela ${ }^{1, *(\mathbb{D})}$ \\ 1 Department of Nutrition, Faculty of Medicine, University of Chile, Santiago 8320000, Chile; \\ rchamorro@uchile.cl (R.C.); kbascunan@uchile.cl (K.A.B.); cbarrera@uchile.cl (C.B.) \\ 2 Obstetrics and Gynecology Department, Clinical Hospital of the University of Chile, Santiago 8320000, Chile \\ josandoval@med.uchile.cl (J.S.); cpuigrredon@hcuch.cl (C.P.) \\ * Correspondence: rvalenzuelab@uchile.cl
}

\section{check for}

updates

Citation: Chamorro, R.; Bascuñán, K.A. Barrera, C.; Sandoval, J.; Puigrredon, C.; Valenzuela, R. Reduced n-3 and n-6 PUFA (DHA and AA)

Concentrations in Breast Milk and Erythrocytes Phospholipids during Pregnancy and Lactation in Women with Obesity. Int. J. Environ. Res. Public Health 2022, 19, 1930. https:/ / doi.org/10.3390/ijerph19041930

Academic Editor: Chiara Ionio

Received: 21 December 2021

Accepted: 2 February 2022

Published: 9 February 2022

Publisher's Note: MDPI stays neutral with regard to jurisdictional claims in published maps and institutional affiliations.

Copyright: (C) 2022 by the authors. Licensee MDPI, Basel, Switzerland. This article is an open access article distributed under the terms and conditions of the Creative Commons Attribution (CC BY) license (https:// creativecommons.org/licenses/by/ $4.0 /)$.

\begin{abstract}
Obesity during pregnancy is a worrying public health problem worldwide. Maternal diet is critical for fatty acid (FA) placental transport and FA content in breast milk (BM). We evaluated FA composition in erythrocytes phospholipids (EP) and BM in pregnant women with (OBE, $\mathrm{n}=30)$ and without (non-OBE, $\mathrm{n}=31$ ) obesity. Sixty-one healthy women were evaluated at their 20-24th gestational week and followed until 6th month of lactation. Diet was evaluated through a food frequency questionnaire. FA composition of EP and BM was assessed by gas-liquid chromatography. The OBE group showed lower diet quality, but total n- 6 and n-3 polyunsaturated FA (PUFA), ALA, EPA, and DHA dietary intake was similar between groups. N-3 PUFA, ALA, DHA, and the n-6/n-3 PUFA ratio in EP were lower at the 6th lactation month in the OBE group. In BM, the arachidonic acid (AA) concentration was lower at the end of the lactation, and DHA content showed an earlier and constant decline in the OBE group compared to the non-OBE group. In conclusion, n-3 PUFA and AA and DHA levels were reduced in EP and BM in pregnant women with obesity. Strategies to increase n-3 PUFA are urgently needed during pregnancy and lactation, particularly in women with obesity.
\end{abstract}

Keywords: obesity; pregnancy; lactation; maternal diet; breast milk; arachidonic acid; docosahexaenoic acid

\section{Introduction}

Maternal nutrition during pregnancy and lactation is relevant for fetal and infant human development [1]. An adequate nutrient supply from mother-to-fetus and motherto-newborn is critical for short- and long-term health outcomes of offspring [2,3]. Maternal diet and nutrition before conception, during pregnancy and breastfeeding, are essential to ensure adequate nutrients supply to the fetus and infant [4]. The association of perinatal maternal morbidity with preconception obesity has been well established. The effects of maternal obesity in utero range from fetal growth, neonatal body composition, and later adolescent obesity [5,6].

Obesity during pregnancy is a rising public health problem in different societies [6] and is rapidly increasing worldwide [7-10]. Excessive weight gain in this period is common (about 50\% of pregnant women) [9] and is consistently associated with adverse fetal and child's metabolic and neurocognitive outcomes [11,12]. Moreover, a higher risk for macrosomia, newborns large for gestational age, cesarean delivery, and maternal hypertension alterations have been reported $[13,14]$. Obesity is common during pregnancy, affecting both the mother and her offspring [15]. It causes short- and long-term problems for the mother, such as the increased risk of excessive gestational weight gain (GWG), gestational diabetes 
(GDM), pre-eclampsia, and cardiovascular diseases [16]. In the offspring, maternal obesity increases prematurity risk, fetal death, injury during birth, transient respiratory problems, and metabolic effects (i.e., insulin resistance in utero [17] and neonatal hypoglycemia) [18]. Maternal obesity also predisposes the offspring to long-term health problems, potentially generating an intergenerational cycle of obesity and insulin resistance $[19,20]$.

Diet plays a critical role during pregnancy, and a high energy intake through highsugar and high-saturated fat foods (energy-dense foods) contribute to excessive weight gain through this period [4]. During breastfeeding, maternal diet and body stores accumulated during pregnancy will influence-among others-the fatty acids (FA) composition of breast milk, particularly the content of n-6 and n-3 polyunsaturated fatty acids (PUFA) [21]. These FA have a critical role in human biochemistry and physiology [22]. In breast milk, arachidonic (C20:4n-6, AA) and docosahexaenoic (C22:6n-3, DHA) acids are two of the most important PUFA for infant development $[23,24]$. Specifically, DHA and AA give flexibility and fluidity to the neuronal plasma membrane, facilitating the signal transduction process, neuronal growth and migration, synaptogenesis, and synaptic plasticity [25]. In addition, these PUFA can modulate energetic metabolism in muscle and adipose tissue, such as insulin action and inflammatory response [26].

The supply of AA and DHA to the fetus and the newborn can originate from: (i) mother's FA stores, (ii) endogenous maternal synthesis or (iii) directly from the maternal diet [27]. Obesity during pregnancy and lactation could lead to (i) decreased hepatic PUFA synthesis [28] or (ii) reduced circulating PUFA levels [29]. It has been demonstrated that placental uptake of PUFA (linoleic acid and DHA) was lower in women with obesity, suggesting a reduced capacity for materno-fetal long-chain PUFA transfers in this group of women [30]. We have reported that the maternal diet of healthy women during pregnancy and breastfeeding characterizes by a deficient intake of n-3 PUFA (especially DHA) [31] This dietary insufficiency was reflected in decreased DHA content of breast milk at 6 th postpartum month [31]. Here, we aimed to evaluate FA composition in erythrocytes (during pregnancy and lactation) and breast milk (from first to sixth months of lactation) in pregnant women with (OBE) and without obesity (non-OBE).

\section{Material and Methods}

\subsection{Study Design and Participants}

This is an observational study including sixty-one pregnant women attending at the Obstetrical and Gynecology Unit, Clinical Hospital of the University of Chile. We included adult pregnant women with an age range between 20 and 35 years, gestational age between 22 and 24 weeks (according to the date of the last menstrual period and confirmed by ultrasound), with a previously successful and healthy pregnancy (having a single term and no more than two newborns) and having successful lactation in previous pregnancies. As exclusion criteria, we considered women with a history of drugs or alcohol consumption, a regular intake of PUFA supplements, being underweight (defined by the Chilean charts for pregnant women [32]), having experienced a previous twin pregnancy, chronic diseases, such as type 2 diabetes, hypertension, and any illness or medical condition that could affect normal fetal growth.

The study protocol was reviewed and approved by the Institutional Review Board of the Faculty of Medicine, University of Chile (Protocol \#073-2011) and by the Ethics Committee of the Clinical Hospital, University of Chile (Protocol \#507/11). All information about the study was given to each participant who voluntarily agreed to participate. Women read and signed the written informed consent.

\subsection{Clinical and Nutritional Evaluation}

When incorporated into the study, women underwent a clinical evaluation when incorporated into the study ( 22 gestational weeks). A physician and a trained nurse evaluated each woman as part of the regular health controls following the national clinical protocol. Bodyweight $(\mathrm{kg})$ and height $(\mathrm{m})$ were evaluated twice with women using light 
clothes, and body-mass index (BMI, $\mathrm{kg} / \mathrm{m}^{2}$ ) was then calculated. Maternal nutritional status was evaluated using BMI according to gestational weeks [32]. The use of the body-mass index (BMI) was validated at the national level to assess pregnant women's nutritional status. In brief, Chilean charts for pregnant women adjust BMI classification according to gestational weeks. The chart validated the use of BMI cut-off points in the context of gestational weight gain data associated with a lower risk of maternal and fetal morbidity [32].

Two groups of pregnant women were studied: a group with obesity $(\mathrm{OBE}, \mathrm{n}=30)$ and a group of normal-weight women (non-OBE, $\mathrm{n}=31$ ) according to gestational weeks [32] Energy and nutrient requirements were calculated following WHO criteria [33] and recommended daily dietary intake followed the American Institute of Medicine criteria (2001) [34]. All women underwent a complete nutritional assessment, including nutritional diagnosis and counseling for a healthy diet following national dietary guidelines [35].

\subsection{Dietary Intake}

Dietary intake was evaluated through a food-frequency questionnaire (FFQ) applied by two trained nutritionists. FFQ was applied at the first clinical evaluation (between 20 and 24 th gestational week), during the first week after delivery, and at the 6th lactation month. Each woman was interviewed and asked to report all foods consumed during the last month. Nutritionists used the "Atlas of Commonly Consumed Foods in Chile", a photographic tool for a precise estimation of serving portions of each food/beverage [36]. Dietary data were checked by contrasting energy/nutrient intake data with dietary questionnaires, identifying missing information and potential outliers. A careful review of each FFQ was done, and, if needed, participants were contacted, and data was checked.

According to the dietary analysis previously described by our workgroup [37], data were grouped into nine food groups (cereals, fruits and vegetables, dairy, meats and eggs, legumes, fish and shellfish, high-lipid foods, oils and fats, and sugars and processed foods). Daily consumption of each reported food/beverage was calculated, and dietary composition was analyzed using Food Processor SQL ${ }^{\circledR}$ (ESHA Research, Salem, OR, USA). We also used a locally generated database, including nutrient composition data from manufacturer labeling of foods and preparations commonly used in Chile. Energy and nutrient intake were estimated using a food database from the USDA National Nutrient Database for Standard Reference.

\subsection{Blood and Breast Milk Samples}

Blood samples were obtained at the 6th month of pregnancy, and the 1st and 6th month of lactation. Butylated hydroxytoluene (BHT) was added to blood samples as an antioxidant, and samples were immediately centrifuged. The erythrocyte fraction was obtained $\left(3000 \times g\right.$ for $10 \mathrm{~min}$ at $\left.20^{\circ} \mathrm{C}\right)$. After, samples were frozen at $-80^{\circ} \mathrm{C}$ for further analysis. During the first six months of lactation, breast milk $(5 \mathrm{~mL})$ samples were obtained monthly and collected in plastic vials after newborns had been fed for at least 2 min; samples were immediately frozen at $-80^{\circ} \mathrm{C}$ for further analysis.

\subsection{Lipids Extraction from Erythrocytes and Breast Milk}

Quantitative extraction of total lipids from erythrocytes phospholipids and breast milk was carried out according to Bligh and Dyer [38], with the addition of Butylated hydroxytoluene (BHT). Erythrocytes and breast milk samples were mixed separately with ice-cold chloroform/methanol (2:1 v/v, containing 0.01\% BHT). Magnesium chloride was added $(0.5 \mathrm{~N})$ and the mixture homogenized in an Ultraturrax homogenizer (Janke \& Kunkel, Stufen, Germany). Total lipids from erythrocytes and milk samples were separated by thin-layer chromatography (TLC), as reported previously [39]. 


\subsection{Gas Chromatography Analysis of Fatty Acid Methyl Esters}

Fatty acid methyl esters (FAMEs) from erythrocyte phospholipids and milk FA were prepared according to Morrison and Smith [40]. Samples previously dissolved in chloroform/methanol $(2: 1 \mathrm{v} / \mathrm{v})$ were then evaporated under nitrogen stream until the volume was halved. Boron trifluoride (12\% methanolic solution) and sodium hydroxide ( $0.5 \mathrm{~N}$ methanolic solution) were added, after which the mixture was cooled. FAMEs were extracted with $0.5 \mathrm{~mL}$ of hexane. Details of the gas chromatographic analysis of FA from erythrocyte phospholipids and breast milk samples were previously described by Valenzuela et al. [41]. FA level was expressed as moles \%.

\subsection{Statistical Analysis}

After descriptive analysis, the distribution of variables was evaluated using the Shapiro-Wilk test and visual inspection. Assessment of significant differences between groups regarding diet and FA composition were evaluated using Student's $t$-test for unpaired data or one-way analysis of variance (ANOVA), with Bonferroni as post hoc test $(p<0.05)$. The association between DHA and AA concentration in breast milk samples and BMI was evaluated through Pearson's correlation test. Data are expressed as mean \pm SD. Statistical significance was set at a 5\% alpha level. The software SPSS v.24.0 (Chicago, IL, USA) was used in all analyses.

\section{Results}

\subsection{Background Characteristics}

Table 1 shows the main background characteristics of participants. Women were mainly young (age range between 20 and 35 years). As expected by the study design, preconception weight and BMI were higher in the obese group. GWG, estimated as the difference between body weight at delivery and preconception weight (in $\mathrm{kg}$ ), was not different between groups. Anthropometrical differences remain significant between groups at the 6th pregnancy month and at the 1st and 6th lactation months. Gestational weeks showed no differences between groups during pregnancy and at delivery.

Table 1. Background characteristics.

\begin{tabular}{cccc}
\hline & $\begin{array}{c}\text { Non-OBE } \\
(\mathbf{n}=\mathbf{3 1})\end{array}$ & $\begin{array}{c}\text { OBE } \\
(\mathbf{n}=\mathbf{3 0})\end{array}$ & $\boldsymbol{p}$-Value \\
\hline Age (years) & $28.5 \pm 4.5$ & $27.1 \pm 5.2$ & 0.784 \\
Preconception weight $(\mathrm{kg})$ & $54.7 \pm 4.7$ & $76.3 \pm 6.6$ & $0.031^{*}$ \\
Height $(\mathrm{m})$ & $1.56 \pm 0.15$ & $1.62 \pm 0.17$ & 0.684 \\
Preconception BMI $\left(\mathrm{kg} / \mathrm{m}^{2}\right)$ & $22.6 \pm 2.2$ & $29.1 \pm 2.9$ & $0.039^{*}$ \\
\hline At enrollment & & & \\
Weight $(\mathrm{kg})$ & $62.1 \pm 5.5$ & $84.9 \pm 7.4$ & $0.042^{*}$ \\
BMI $\left(\mathrm{kg} / \mathrm{m}^{2}\right)$ & $25.5 \pm 2.4$ & $32.5 \pm 2.8$ & $0.031^{*}$ \\
Gestational age $(\mathrm{weeks})$ & $24.5 \pm 1.9$ & $24.9 \pm 2.2$ & 0.814 \\
\hline After delivery & & & \\
Weight $(\mathrm{kg})$ & $66.8 \pm 6.9$ & $89.8 \pm 7.2$ & $0.035^{*}$ \\
BMI $\left(\mathrm{kg} / \mathrm{m}^{2}\right)$ & $27.8 \pm 1.9$ & $34.2 \pm 2.8$ & 0.043 * \\
Gestational age $($ weeks) & $39.1 \pm 2.2$ & $37.9 \pm 2.4$ & 0.793 \\
Body weight gain $(\mathrm{kg}){ }^{\dagger}$ & $12.2 \pm 4.7$ & $13.6 \pm 5.9$ & 0.629 \\
\hline At 6th postpartum month & & & \\
Weight $(\mathrm{kg})$ & $57.6 \pm 4.9$ & $83.7 \pm 6.8$ & $0.029 *$ \\
BMI $\left(\mathrm{kg} / \mathrm{m}^{2}\right)$ & $23.9 \pm 1.8$ & $32.3 \pm 3.0$ & $0.037^{*}$ \\
\hline
\end{tabular}

Data are shown as mean $\pm \mathrm{SD}$, or as a percentage (\%); SES, socioeconomic status; BMI, body mass index $=\mathrm{kg} / \mathrm{m}^{2}$; non-OBE: women without obesity; OBE: women with obesity. All data showed normal distribution. Groups were compared with Student's $t$-test for independent samples $(p<0.05) .{ }^{*}$ Indicates statistically significant differences.

${ }^{+}$Body weight gain (weight at delivery-preconception weight). 


\subsection{Food Groups Consumption}

Comparison of food groups consumption between OBE and non-OBE groups showed several differences. Lower fruits/vegetables and dairy intake were observed in the OBE group during all evaluated periods (Table 2). The same group also showed higher consumption of high-fat foods, oils and fats, and sugar and processed foods. No between-groups differences were observed regarding other food groups (Table 2).

Table 2. Daily food groups intake (g/day) across pregnancy and lactation time points.

\begin{tabular}{|c|c|c|c|c|c|c|}
\hline \multirow[b]{2}{*}{ Food Groups } & \multicolumn{2}{|c|}{ 6th Pregnancy Month } & \multicolumn{2}{|c|}{ 1st Lactation Month } & \multicolumn{2}{|c|}{ 6th Lactation Month } \\
\hline & Non-OBE ${ }^{a}$ & $\mathrm{OBE}^{\mathbf{b}}$ & Non-OBE ${ }^{c}$ & $\mathrm{OBE}^{\mathrm{d}}$ & Non-OBE ${ }^{\mathrm{e}}$ & OBE $^{f}$ \\
\hline Cereals & $298.2 \pm 32.4$ & $382.3 \pm 51.0$ & $284.7 \pm 35.8$ & $372.5 \pm 45.2$ & $271.7 \pm 39.5$ & $366.3 \pm 50.4$ \\
\hline Fruits and Vegetables & $501.5 \pm 47.6^{\mathrm{b}, \mathrm{d}, \mathrm{f}}$ & $375.2 \pm 32.9^{a, c, e}$ & $472.6 \pm 58.9^{\mathrm{b}, \mathrm{d}, \mathrm{f}}$ & $302.9 \pm 31.4^{\mathrm{a}, \mathrm{c}, \mathrm{e}}$ & $457.8 \pm 38.9^{\mathrm{b}, \mathrm{d}, \mathrm{f}}$ & $314.5 \pm 25.9^{\mathrm{a}, \mathrm{c}, \mathrm{e}}$ \\
\hline Dairy & $599.7 \pm 40.9^{\mathrm{b}, \mathrm{d}, \mathrm{f}}$ & $325.6 \pm 31.9^{a, c, e}$ & $492.7 \pm 42.6^{\mathrm{b}, \mathrm{d}, \mathrm{f}}$ & $294.5 \pm 29.9^{a, c, e}$ & $479.2 \pm 45.2^{\mathrm{b}, \mathrm{d}, \mathrm{f}}$ & $239.4 \pm 50.6^{\mathrm{a}, \mathrm{c}, \mathrm{e}}$ \\
\hline Meats and Eggs & $101.1 \pm 12.5$ & $119.4 \pm 29.9$ & $89.5 \pm 19.6$ & $107.6 \pm 19.4$ & $85.9 \pm 10.8$ & $102.8 \pm 26.3$ \\
\hline Fish and Seafood & $25.3 \pm 6.7$ & $22.1 \pm 6.0$ & $28.9 \pm 7.4$ & $24.5 \pm 10.5$ & $23.5 \pm 5.5$ & $20.4 \pm 6.9$ \\
\hline Legumes & $30.1 \pm 8.5$ & $18.2 \pm 9.6$ & $25.9 \pm 7.1$ & $15.4 \pm 7.5$ & $35.8 \pm 10.2$ & $20.1 \pm 5.8$ \\
\hline High-Fat Foods & $25.2 \pm 5.8^{\mathrm{b}, \mathrm{d}, \mathrm{f}}$ & $56.9 \pm 10.6^{\mathrm{a}, \mathrm{c}, \mathrm{e}}$ & $23.6 \pm 6.3^{\mathrm{b}, \mathrm{d}, \mathrm{f}}$ & $50.1 \pm 13.6^{\mathrm{a}, \mathrm{c}, \mathrm{e}}$ & $20.9 \pm 5.5^{\mathrm{b}, \mathrm{d}, \mathrm{f}}$ & $52.8 \pm 9.8^{a, c, e}$ \\
\hline Oils and Fats & $28.9 \pm 6.3^{\mathrm{b}, \mathrm{d}, \mathrm{f}}$ & $44.6 \pm 9.6^{\mathrm{a}, \mathrm{c}, \mathrm{e}}$ & $25.9 \pm 5.4^{\mathrm{b}, \mathrm{d}, \mathrm{f}}$ & $40.8 \pm 10.6^{\mathrm{a}, \mathrm{c}, \mathrm{e}}$ & $23.7 \pm 8.2^{\mathrm{b}, \mathrm{d}, \mathrm{f}}$ & $41.8 \pm 12.6^{\mathrm{a}, \mathrm{c}, \mathrm{e}}$ \\
\hline $\begin{array}{c}\text { Sugar and Processed } \\
\text { Foods }\end{array}$ & $161.4 \pm 30.5^{\mathrm{b}, \mathrm{d}, \mathrm{f}}$ & $402.6 \pm 69.7^{\mathrm{a}, \mathrm{c}, \mathrm{e}}$ & $182.6 \pm 44.6^{\mathrm{b}, \mathrm{d}, \mathrm{f}}$ & $384.5 \pm 60.1^{\mathrm{a}, \mathrm{c}, \mathrm{e}}$ & $170.5 \pm 38.6^{\mathrm{b}, \mathrm{d}, \mathrm{f}}$ & $371.5 \pm 78.3^{\mathrm{a}, \mathrm{c}, \mathrm{e}}$ \\
\hline
\end{tabular}

Data are mean \pm SD. a Significantly different to normal weight 6 th month of pregnancy; ${ }^{b}$ Significantly different to obese 6th month of pregnancy; ${ }^{\mathrm{C}}$ Significantly different to normal weight 1 st month of lactation; ${ }^{\mathrm{d}}$ Significantly

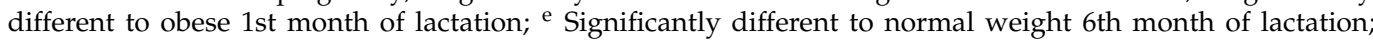
${ }^{\mathrm{f}}$ Significantly different to obese 6 th month of lactation. Food intake was organized in nine groups according to the methodology described in the text (see methods). One-way ANOVA and Bonferroni test $(p<0.05)$. non-OBE: women without obesity; OBE: women with obesity.

\subsection{Energy, Nutrients, and FA Dietary Intake}

Table 3 shows energy, nutrients, and the most relevant fatty acids dietary intake. The OBE group showed consistently higher energy intake across all three periods than the non-OBE group. Total protein and carbohydrate intake were similar, but the OBE group's sugar intake was higher. In all evaluated periods, total fat intake was significantly higher in the OBE group, mainly due to a higher saturated fatty acids (SFA) intake (Table 3). Total n-6 and n-3 PUFA and alpha-linolenic acid (C18:3n-3, ALA), eicosapentaenoic acid (C20:5n-3, EPA), and DHA intake were similar between groups.

\subsection{Fatty Acid Composition of Erythrocyte Phospholipids}

Table 4 shows the FA composition of erythrocyte phospholipids. No significant differences were found for SFA, monounsaturated fatty acids (MUFA), PUFA, n-6 PUFA, linoleic acid (C18:2n-6, LA), and n-6 docosapentaenoic acid (C22:5n-6, DPAn-6) n-6 when comparing FA concentration between the 1st lactation month and the 6th lactation between OBE and non-OBE groups. However, AA concentration was reduced in the OBE group at the 6th lactation month. N-3 PUFA, ALA, DHA, and the n-6/n-3 PUFA ratio was significantly lower in the OBE group for all studied periods. In addition, EPA and docosapentaenoic acid (C22:5n-3, DPAn-3) showed reduced levels in the OBE group, particularly at the 6th lactation month (Table 4). 
Table 3. Energy, nutrients, and most relevant fatty acid intake during pregnancy and lactation periods.

\begin{tabular}{|c|c|c|c|c|c|c|}
\hline \multirow[b]{2}{*}{$\begin{array}{c}\text { Energy/Nutrients/Fatty } \\
\text { Acid }\end{array}$} & \multicolumn{2}{|c|}{ 6th Month of Pregnancy } & \multicolumn{2}{|c|}{ 1st Month of Lactation } & \multicolumn{2}{|c|}{ 6th Month of Lactation } \\
\hline & Non-OBE ${ }^{\text {a }}$ & $\mathrm{OBE}^{\mathrm{b}}$ & Non-OBE ${ }^{c}$ & OBE $^{d}$ & Non-OBE ${ }^{e}$ & $\mathrm{OBE}^{\mathrm{f}}$ \\
\hline Energy (kcal/day) & $2142 \pm 274^{b}$ & $2895 \pm 316^{\mathrm{a}, \mathrm{c}, \mathrm{e}}$ & $1852 \pm 205^{\mathrm{b}, \mathrm{d}, \mathrm{f}}$ & $2659 \pm 302^{c, e}$ & $1793 \pm 199^{\mathrm{a}, \mathrm{b}, \mathrm{d}, \mathrm{f}}$ & $2438 \pm 294^{c, e}$ \\
\hline Protein (g/day) & $81.1 \pm 26.5$ & $98.5 \pm 31.5$ & $73.1 \pm 24.3$ & $90.6 \pm 29.4$ & $66.5 \pm 19.2$ & $84.5 \pm 22.3$ \\
\hline Carbohydrate (g/day) & $325.3 \pm 62.4$ & $449.5 \pm 72.3$ & $247.2 \pm 51.4$ & $401.6 \pm 82.9$ & $232.1 \pm 44.6$ & $362.4 \pm 80.1$ \\
\hline Fiber (g/day) & $42.7 \pm 14.9^{\mathrm{f}}$ & $20.1 \pm 10.3$ & $39.6 \pm 12.8$ & $18.5 \pm 9.3$ & $31.7 \pm 9.2$ & $17.2 \pm 6.8^{\mathrm{a}}$ \\
\hline Sugar (g/day) & $90.5 \pm 20.5^{b, d, f}$ & $198.5 \pm 45.9^{a, c, e}$ & $82.6 \pm 22.9^{b, d, f}$ & $184.3 \pm 38.7^{\mathrm{a}, \mathrm{c}, \mathrm{e}}$ & $72.4 \pm 18.0^{\mathrm{b}, \mathrm{d}, \mathrm{f}}$ & $175.2 \pm 32.6^{\mathrm{a}, \mathrm{c}, \mathrm{e}}$ \\
\hline Fat (g/day) & $74.8 \pm 11.4^{\mathrm{b}, \mathrm{d}, \mathrm{f}}$ & $124.1 \pm 21.3^{\mathrm{a}, \mathrm{c}, \mathrm{e}}$ & $69.2 \pm 14.1^{\mathrm{b}, \mathrm{d}, \mathrm{f}}$ & $121.3 \pm 19.1^{\mathrm{a}, \mathrm{c}, \mathrm{e}}$ & $69.6 \pm 15.8^{b, d, f}$ & $115.5 \pm 22.0^{\mathrm{a}, \mathrm{c}, \mathrm{e}}$ \\
\hline$\Sigma$ SFA (g/day) & $20.1 \pm 2.5^{\mathrm{b}, \mathrm{d}, \mathrm{f}}$ & $44.3 \pm 3.8^{\mathrm{a}, \mathrm{c}, \mathrm{e}}$ & $18.7 \pm 2.2^{\mathrm{b}, \mathrm{d}, \mathrm{f}}$ & $36.9 \pm 3.6^{\mathrm{a}, \mathrm{c}, \mathrm{e}}$ & $16.5 \pm 2.2^{\mathrm{b}, \mathrm{d}, \mathrm{f}}$ & $32.5 \pm 3.3^{\mathrm{a}, \mathrm{c}, \mathrm{e}}$ \\
\hline$\Sigma$ MUFA (g/day) & $27.6 \pm 3.6$ & $35.7 \pm 3.0$ & $25.9 \pm 2.7$ & $38.9 \pm 2.9$ & $25.1 \pm 2.4$ & $37.7 \pm 2.5$ \\
\hline इPUFA (g/day) & $26.9 \pm 2.9$ & $42.1 \pm 4.1^{\mathrm{a}, \mathrm{c}, \mathrm{e}}$ & $24.6 \pm 2.5$ & $45.4 \pm 6.7^{\mathrm{a}, \mathrm{c}, \mathrm{e}}$ & $27.9 \pm 2.8$ & $45.1 \pm 6.4^{\mathrm{a}, \mathrm{c}, \mathrm{e}}$ \\
\hline$\Sigma n-6$ PUFA (g/day) & $24.8 \pm 2.3$ & $38.0 \pm 3.5^{\mathrm{a}, \mathrm{c}, \mathrm{e}}$ & $21.2 \pm 2.1$ & $41.9 \pm 5.1^{\mathrm{a}, \mathrm{c}, \mathrm{e}}$ & $25.9 \pm 2.5$ & $42.2 \pm 4.2^{\mathrm{a}, \mathrm{c}, \mathrm{e}}$ \\
\hline $18: 2 n-6$ (LA) (g/day) & $23.2 \pm 2.1$ & $36.4 \pm 3.4^{\mathrm{a}, \mathrm{c}, \mathrm{e}}$ & $21.3 \pm 2.3$ & $39.9 \pm 3.7^{a, c, e}$ & $24.7 \pm 2.2$ & $40.8 \pm 2.0^{\mathrm{a}, \mathrm{c}, \mathrm{e}}$ \\
\hline $20: 4 n-6$ (AA) (g/day) & $0.72 \pm 0.2$ & $0.86 \pm 0.3$ & $0.81 \pm 0.2$ & $0.71 \pm 0.1$ & $0.82 \pm 0.2$ & $0.77 \pm 0.1$ \\
\hline$\sum n-3$ PUFA (g/day) & $2.05 \pm 0.6$ & $2.82 \pm 0.7$ & $1.95 \pm 0.5$ & $2.92 \pm 0.8$ & $1.92 \pm 0.5$ & $2.75 \pm 0.4$ \\
\hline $18: 3 n-3$ (ALA) (g/day) & $1.82 \pm 0.6$ & $2.65 \pm 0.7$ & $1.71 \pm 0.4$ & $2.75 \pm 0.7$ & $1.71 \pm 0.6$ & $2.59 \pm 0.4$ \\
\hline $20: 5 n-3$ (EPA) (g/day) & $0.05 \pm 0.02$ & $0.04 \pm 0.01$ & $0.06 \pm 0.02$ & $0.04 \pm 0.01$ & $0.05 \pm 0.02$ & $0.04 \pm 0.02$ \\
\hline 22:6n-3 (DHA) (g/day) & $0.12 \pm 0.03$ & $0.09 \pm 0.2$ & $0.13 \pm 0.04$ & $0.10 \pm 0.02$ & $0.11 \pm 0.03$ & $0.08 \pm 0.02$ \\
\hline$n-6 / n-3$ PUFA ratio & $12.1 \pm 1.2$ & $13.4 \pm 1.4$ & $11.5 \pm 1.1$ & $14.1 \pm 1.8$ & $13.4 \pm 1.4$ & $15.3 \pm 1.9$ \\
\hline
\end{tabular}

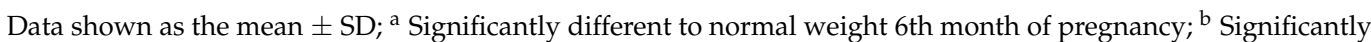
different to obese 6th month of pregnancy; ${ }^{c}$ Significantly different to normal weight 1st month of lactation d Significantly different to obese 1st month of lactation; ${ }^{\text {e }}$ Significantly different to normal weight 6 th month of lactation; ${ }^{\mathrm{f}}$ Significantly different to obese 6th month of lactation. One-way ANOVA and Bonferroni test. $(p<0.05)$. Saturated fatty acids (SFA) correspond to C6:0, C8:0, C10:0, C12:0, C14:0, C16:0, C18:0, C20:0, C22:0, and C24:0. Monounsaturated fatty acids (MUFA) correspond to C14:1n-5, C16:1n-7, and C18:1n-9. Polyunsaturated fatty acids (PUFA) correspond to C18:2n-6, C18:3n-3, C20:4n-6, C20:5n-3, C22:5n-6, C22:5n-3, and 22:6n-3. n-6 PUFA correspond to C18:2n-6, C20:4n-6, and C22:5n-6. n-3 PUFA correspond to C18:3n-3, C20:5n-3, C22:5n-3, and C22:6n-3. non-OBE: women without obesity; OBE: women with obesity.

Table 4. Fatty acid composition in erythrocyte phospholipids of the women during the pregnancy and lactation period.

\begin{tabular}{|c|c|c|c|c|c|c|}
\hline \multirow[b]{2}{*}{$\begin{array}{c}\text { FA (Expressed as mol } \\
\% \text { of Fatty Acid) }\end{array}$} & \multicolumn{2}{|c|}{ 6th Month of Pregnancy } & \multicolumn{2}{|c|}{ 1st Month of Lactation } & \multicolumn{2}{|c|}{ 6th Month of Lactation } \\
\hline & Non-OBE ${ }^{a}$ & $\mathrm{OBE}^{\mathrm{b}}$ & Non-OBE ${ }^{c}$ & OBE $^{d}$ & Non-OBE ${ }^{\mathrm{e}}$ & OBE $^{f}$ \\
\hline$\Sigma S F A$ & $49.8 \pm 4.7$ & $53.7 \pm 5.1$ & $48.8 \pm 5.0$ & $52.9 \pm 5.6$ & $49.3 \pm 5.2$ & $53.0 \pm 5.4$ \\
\hline C16:0 & $30.3 \pm 3.2$ & $33.2 \pm 3.7$ & $30.9 \pm 2.9$ & $32.9 \pm 3.5$ & $31.5 \pm 3.3$ & $34.5 \pm 3.6$ \\
\hline C18:0 & $15.8 \pm 1.8$ & $18.8 \pm 1.9$ & $16.1 \pm 1.4$ & $17.9 \pm 1.9$ & $16.5 \pm 1.4$ & $18.2 \pm 1.9$ \\
\hline$\Sigma$ MUFA & $16.8 \pm 1.7$ & $15.2 \pm 1.4$ & $17.3 \pm 1.6$ & $15.9 \pm 1.7$ & $18.3 \pm 1.8$ & $16.9 \pm 1.5$ \\
\hline $\mathrm{C} 18: 1 n-9$ & $14.5 \pm 1.4$ & $13.8 \pm 1.3$ & $15.5 \pm 1.7$ & $13.6 \pm 1.4$ & $17.3 \pm 1.8$ & $15.5 \pm 1.2$ \\
\hline$\Sigma$ PUFA & $33.4 \pm 3.8$ & $31.3 \pm 3.2$ & $33.9 \pm 3.6$ & $31.2 \pm 3.0$ & $32.4 \pm 3.7$ & $30.1 \pm 3.3$ \\
\hline$\sum n-6$ PUFA & $24.8 \pm 2.5$ & $25.9 \pm 2.9$ & $25.8 \pm 2.4$ & $26.3 \pm 2.7$ & $24.5 \pm 2.6$ & $25.7 \pm 2.8$ \\
\hline C18:2n-6 (LA) & $11.3 \pm 1.3$ & $12.6 \pm 1.5$ & $12.8 \pm 1.9$ & $13.5 \pm 2.2$ & $12.7 \pm 1.4$ & $13.9 \pm 1.7$ \\
\hline C20:4 n-6 (AA) & $12.5 \pm 1.6$ & $11.9 \pm 1.4$ & $11.6 \pm 1.3$ & $11.2 \pm 1.5$ & $10.7 \pm 1.2$ & $9.61 \pm 0.5^{\mathrm{a}}$ \\
\hline C22:5n-6 (DPAn-6) & $0.81 \pm 0.1$ & $0.79 \pm 0.2$ & $0.87 \pm 0.05$ & $0.81 \pm 0.1$ & $0.95 \pm 0.2$ & $0.92 \pm 0.3$ \\
\hline$\sum n-3$ PUFA & $8.60 \pm 1.0^{\mathrm{b}, \mathrm{d}, \mathrm{f}}$ & $5.40 \pm 0.4^{\mathrm{a}, \mathrm{c}, \mathrm{e}}$ & $8.10 \pm 0.9^{\mathrm{b}, \mathrm{d}, \mathrm{f}}$ & $4.90 \pm 0.5^{\mathrm{a}, \mathrm{c}, \mathrm{e}}$ & $7.90 \pm 1.1^{\mathrm{b}, \mathrm{d}, \mathrm{f}}$ & $4.38 \pm 0.4^{\mathrm{a}, \mathrm{c}, \mathrm{e}}$ \\
\hline C18:3n-3 (ALA) & $1.88 \pm 0.3^{\mathrm{b}, \mathrm{d}, \mathrm{f}}$ & $1.15 \pm 0.2^{\mathrm{a}, \mathrm{c}, \mathrm{e}}$ & $2.09 \pm 0.5^{\mathrm{b}, \mathrm{d}, \mathrm{f}}$ & $1.05 \pm 0.3^{\mathrm{a}, \mathrm{c}, \mathrm{e}}$ & $2.15 \pm 0.6^{\mathrm{b}, \mathrm{d}, \mathrm{f}}$ & $1.01 \pm 0.4^{\mathrm{a}, \mathrm{c}, \mathrm{e}}$ \\
\hline C20:5n-3 (EPA) & $1.57 \pm 0.4^{\mathrm{b}, \mathrm{d}, \mathrm{f}}$ & $0.93 \pm 0.1^{\mathrm{a}, \mathrm{d}, \mathrm{f}}$ & $1.24 \pm 0.3^{f}$ & $0.51 \pm 0.2^{\mathrm{a}, \mathrm{b}}$ & $1.19 \pm 0.2^{\mathrm{d}, \mathrm{f}}$ & $0.44 \pm 0.1^{\mathrm{a}, \mathrm{b}, \mathrm{c}, \mathrm{e}}$ \\
\hline $\mathrm{C} 22: 5 n-3(\mathrm{DPA} n-3)$ & $0.58 \pm 0.1^{c, d, e, f}$ & $0.40 \pm 0.1^{\mathrm{d}, \mathrm{e}, \mathrm{f}}$ & $0.38 \pm 0.05^{\mathrm{a}, \mathrm{d}, \mathrm{f}}$ & $0.21 \pm 0.03^{a, b, c}$ & $0.29 \pm 0.04^{\mathrm{a}, \mathrm{b}, \mathrm{f}}$ & $0.17 \pm 0.03^{\mathrm{a}, \mathrm{b}, \mathrm{c}, \mathrm{e}}$ \\
\hline C22:6n-3 (DHA) & $4.53 \pm 0.5^{\mathrm{b}, \mathrm{d}, \mathrm{f}}$ & $3.04 \pm 0.4^{\mathrm{a}, \mathrm{c}, \mathrm{e}}$ & $4.39 \pm 0.6^{\mathrm{b}, \mathrm{d}, \mathrm{f}}$ & $3.01 \pm 0.3^{a, c, e}$ & $4.22 \pm 0.4^{\mathrm{b}, \mathrm{d}, \mathrm{f}}$ & $2.55 \pm 0.3^{a, c, e}$ \\
\hline$n-6 / n-3$ PUFA ratio & $2.87 \pm 0.1^{\mathrm{b}, \mathrm{d}, \mathrm{f}}$ & $4.77 \pm 0.6^{\mathrm{a}, \mathrm{c}, \mathrm{e}}$ & $3.16 \pm 0.5^{\mathrm{b}, \mathrm{d}, \mathrm{f}}$ & $5.34 \pm 0.7^{\mathrm{a}, \mathrm{c}, \mathrm{e}}$ & $3.09 \pm 0.4^{\mathrm{b}, \mathrm{d}, \mathrm{f}}$ & $5.84 \pm 0.6^{\mathrm{a}, \mathrm{c}, \mathrm{e}}$ \\
\hline
\end{tabular}

Data shown as the mean \pm SD and expressed as mol \% of fatty acid. ${ }^{\text {a }}$ Significantly different to normal weight 6 th month of pregnancy; ${ }^{b}$ Significantly different to obese 6 th month of pregnancy; ${ }^{c}$ Significantly different to normal

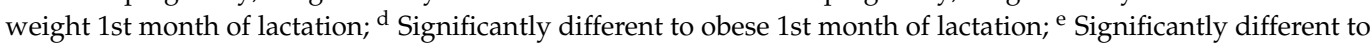

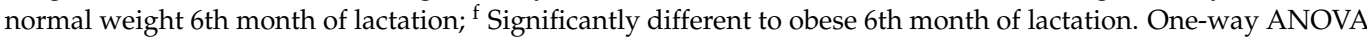
and Bonferroni test. $(p<0.05)$. The description of saturated and unsaturated FA is the same that are shown in legend of Table 3. non-OBE: women without obesity; OBE: women with obesity.

\subsection{Fatty Acid Composition of Breast Milk}

Figures 1 and 2 show the FA composition in breast milk. We found rather similar SFA, MUFA, and total PUFA levels across lactation between both groups (Figure 1A-D). However, n-3 PUFA content at the 4th and 6th lactation months was significantly lower in the OBE group (Figure 1E). N-6 and n-3 fatty acids (LA, ALA, and EPA, Figure 2A,B,D, respectively) concentrations showed similar levels between groups. AA concentration was lower at the OBE group's 5th and 6th lactation months (Figure 2C). Notably, DHA 
concentration in breast milk was significantly lower across the whole lactation period in the OBE group (Figure 2E). DHA content in breast milk showed significant differences across lactation in the OBE group, with a constant and significant DHA decrease (Figure 2E). Starting at the 3rd month, DHA concentration was significantly lower compared with the 1st and 2 nd months $(p<0.05)$. Differences remained until the 6th lactation month, when DHA was still lower than the 1st and 4th months (Figure 2E). The same analysis in the non-OBE group showed reduced DHA content only at months 5 and 6 compared with the previous months (Figure 2E). Finally, correlation analysis showed an inverse association $(\mathrm{r}=-0.83, p<0.0001)$ between DHA (but not AA) content in breast milk and BMI at the 1st lactation month (Figure 3A,B). The same association was found for both DHA ( $\mathrm{r}=-0.91$, $p<0.0001)$ and AA $(r=-0.75, p<0.0001)$ content in breast milk and BMI at the 6th lactation month (Figure 3C,D).

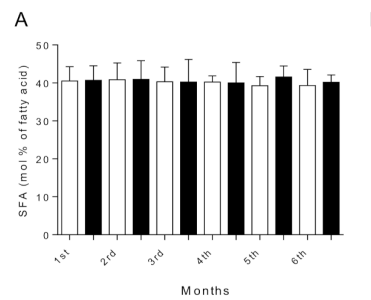

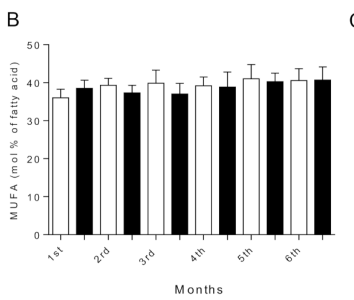

Months
C

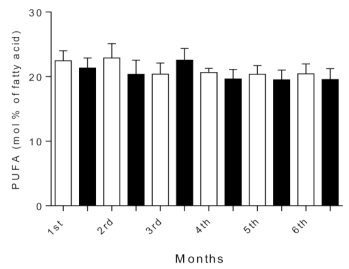

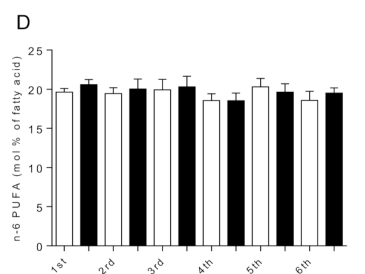

Months

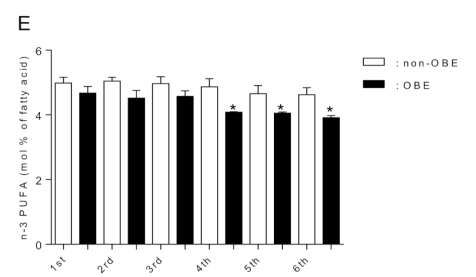

Months

Figure 1. (A) SFA; (B) MUFA; (C) PUFA; (D) n-6 PUFA, and (E) n-3 PUFA content in breast milk. Data as mean $\pm \mathrm{SD}$ and expressed as mol \% of fatty acid (non-OBE, $\mathrm{n}=31$, and $\mathrm{OBE}, \mathrm{n}=30$ ); one-way ANOVA with Bonferroni post hoc test. Columns without a symbol or that share the same character are not significantly different from each other. * Indicates statistically significant differences with the other groups. Non-OBE: women without obesity; OBE: women with obesity.

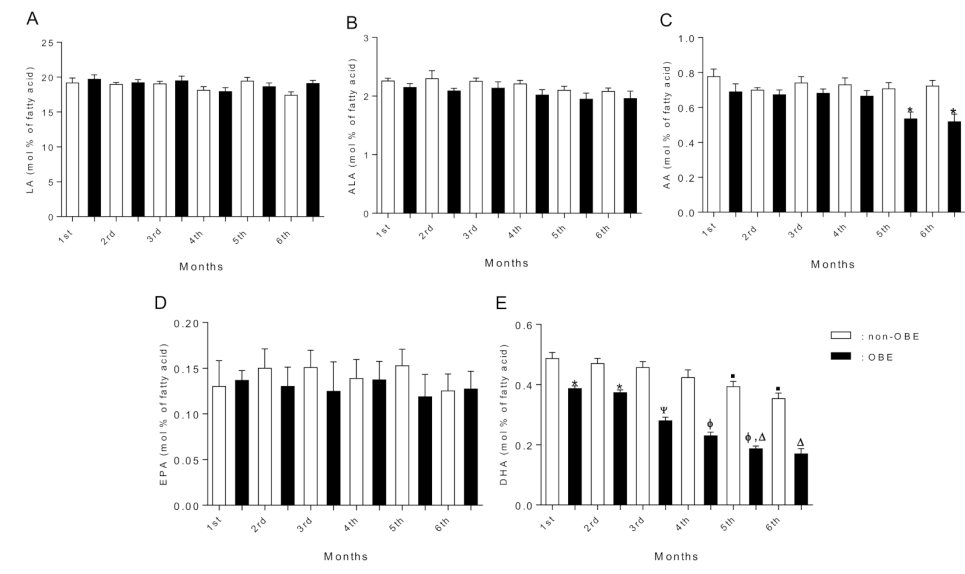

Figure 2. (A) LA; (B) ALA; (C) AA; (D) EPA, and (E) DHA content in breast milk. Data as mean $\pm \mathrm{SD}$ and expressed as mol \% of fatty acid (non-OBE, $\mathrm{n}=31$, and OBE, $\mathrm{n}=30$ ); one-way ANOVA with Bonferroni post hoc test. Columns without a symbol or that share the same character are not significantly different from each other. ${ }^{*}, \Psi, \Phi, \cdot$ or $\Delta$ symbols Indicates statistically significant differences with the other groups. Non-OBE: women without obesity; OBE: women with obesity. 

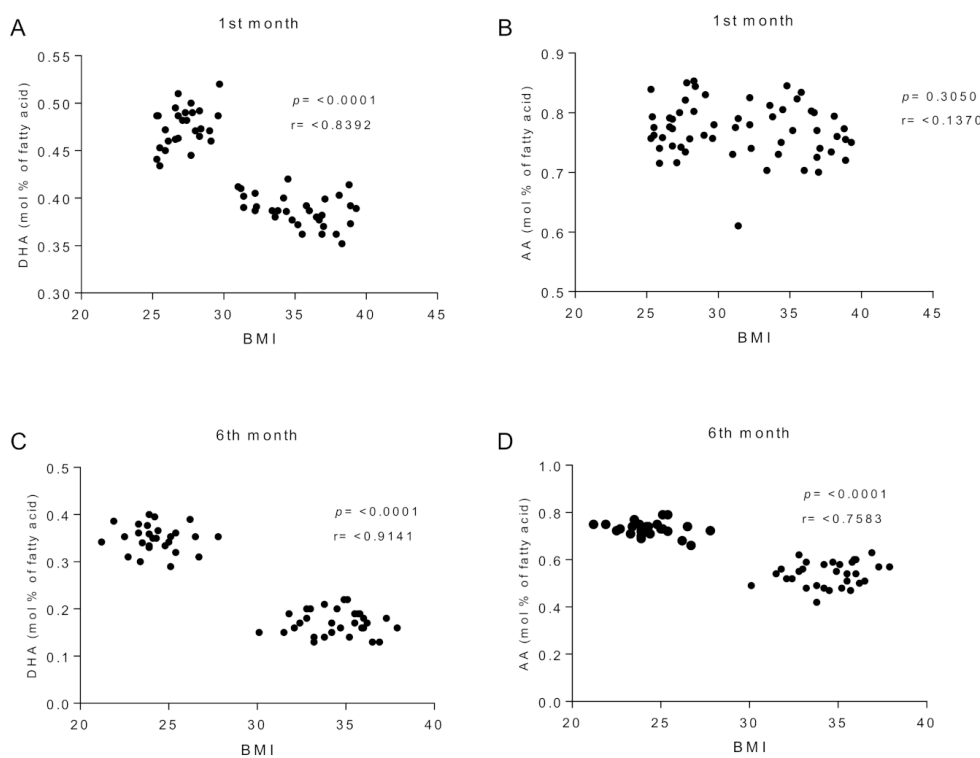

Figure 3. Correlations between DHA and AA breast milk content at 1st and 6th months with bodymass index (BMI). (A) DHA content v/s BMI at 1st lactation month; (B) AA content v/s BMI at 1st lactation month; (C) DHA content v/s BMI at 6th lactation month; (D) AA content v/s BMI at 6th lactation month.

\section{Discussion}

We aimed to evaluate FA composition in erythrocytes and breast milk in a group of pregnant women with and without obesity from the last trimester of pregnancy until the 6th lactation month. Our main findings show that the content of n-3 PUFA and DHA in erythrocyte phospholipids was significantly lower during both pregnancy and lactation in women with obesity. The content of AA and EPA in erythrocyte phospholipids was also reduced at the 6th lactation month in this group. In addition, n-3 PUFA and AA content in breast milk was reduced at the 5th and 6th lactation months in the OBE group. DHA content was consistently lower across the whole lactation period in women with obesity compared with their normal-weight peers.

The worldwide prevalence of obesity has been rising during the last decades. A significant increase in overweight and obesity among pregnant women has been reported in both high-income and middle-income countries [8,42]. In this context, studies suggest that Chilean childbearing women show a fast and growing tendency towards obesity [43], and a worryingly high prevalence of pregnant women with overweight and obesity was observed [44]. Moreover, maternal obesity is related to several health consequences for the mother and infant [12,13], increasing the infant's risk of developing several chronic diseases such as obesity, type 2 diabetes, and cardiovascular diseases [45], and increasing body fat in male 2-6 years children [46]. Our results show that women with obesity displayed an impaired FA profile in erythrocytes and breast milk-compared to a group of normal-weight women-during the last part of pregnancy and the first six months of lactation.

Concerning FA metabolism, obesity during pregnancy poses several adverse consequences, as well. Studies have shown an imbalance in dietary intake of n- 6 and n-3 PUFA in women with obesity mainly increased n-6 PUFA and decreased n-3 PUFA [31,47]. On the other hand, maternal obesity during pregnancy and lactation can alter PUFA metabolism, leading to decreased PUFA levels and synthesis $[28,29]$ and impaired capacity for maternofetal long-chain PUFA transfer [30]. It has also been shown that placental transfer of FA transporters is impaired by obesity [48], leading to reduced PUFA supply to the fetus [30]. The endogenous synthesis of DHA from ALA can be affected by obesity itself and obesity-related metabolic diseases [49], reducing the activity of $\Delta-5$ and $\Delta-6$ desaturase 
enzymes [28], leading to impaired DHA synthesis and lower content of n-3 long-chain PUFA in erythrocytes and breast milk. In this regard, the PUFA synthesis process is directly related to $\Delta-6$ and $\Delta-5$ desaturase enzymes activity (particularly in the liver) [50]. This enzymatic pathway process can be modulated by liver steatosis induced by a high-fat diet (with high SFA content, such as palmitic acid) or oxidative stress [49,50], common metabolic conditions found in obesity [49]. In line with our results, the lower n-3 PUFA, DHA, and AA levels in erythrocytes and breast milk in the OBE group, could be explained by the alteration in the FA desaturation capacity. This is supported by the similar dietary intake of AA, ALA, DHA, and EPA between non-OBE and OBE groups and by the fact that the OBE group showed higher SFA intake than their normal-weight peers (Table 3).

Breast milk provides several nutrients, immune cells, and bioactive molecules during the lactation period for optimal infant growth [51,52]. An adequate supply of DHA and AA through breastfeeding is of paramount relevance for an infant's nutrition and development [53]. DHA and AA content in human milk depends on maternal diet [54-56]. In this regard, humans can synthesize PUFA from LA and ALA, both being essential precursors of more unsaturated and long-chain FA [57]. LA is the precursor of AA [58], whereas ALA is the precursor of EPA and DHA, two of the most representative FA of the n-3 PUFA family [58]. The synthesis of PUFA is an important process that occurs mainly in the liver and provides a constant flow of these FA to cells and tissues, such as the brain, retina, and immune system cells [59]. PUFA synthesis is dependent on the availability of substrate (LA or ALA), activity of the elongases and desaturases enzymes (e.g., $\Delta-5$ and $\Delta-6$ desaturases), availability of specific nutrients and the redox state (particularly in the liver) [59-61].

The relevance of preventing obesity during pregnancy is highlighted by several lines of evidence showing a significant impact on the fetus and infant development. Studies implementing PUFA supplementation have shown positive results improving FA maternal status [25] but more inconsistent results concerning metabolic regulation and infant body composition $[62,63]$. In this regard, the low intake of n-3 long-chain PUFA, as seen in our study, is critical for nutritional intervention. Dietary strategies have been implemented to improve women's diet quality during pregnancy and lactation through educational programs focused on promoting the consumption of foods that provide DHA [64,65]. It is known that it is not easy to modify dietary habits, but pregnancy is when dietary habit modifications can achieve improved results [9]. Several studies have used nutritional supplements containing DHA or DHA-added foods, evidencing that an increase in the intake of DHA increases the content of this FA in erythrocytes and subsequently in breast milk in pregnant and lactating women [66,67]. Therefore, dietary strategies focused on increasing weekly fish consumption can improve maternal PUFA status with potential benefits for the fetus and the infant. However, when obesity is present, the results from these interventions can be attenuated, as women with obesity show a lower increase in n-3 FA levels and a lower reduction in the n-6:n-3 ratio after supplementation compared with normal-weight women [68].

As limitations, we assessed dietary intake using dietary surveys (FFQ), a method that possesses limitations [69]. However, we have used this instrument previously, and it has been validated in this population with the focus on dietary sources of fat and fatty acid intake $[31,37,41]$. We used a second instrument (24-h recall) to corroborate dietary structure and the dietary pattern of studied women, complementing the data from the FFQ. The fact that food information from dietary surveys is based on food composition data is another plausible limitation [70]. Several factors have been reported to potentially affect food composition data, including food sampling processes, as well as sources of analytical, environmental, and biological variability $[70,71]$. We used the USDA National Nutrient Database for reference and included manufacturer and local food composition data for specific national products. This last point allowed us to complement nutritional information with local foods commonly consumed by the Chilean population. Regarding strengths, we can mention that we carried out a complete analysis of the composition of FA in erythrocytes and breast milk, both components that provide complementary information regarding the 
FA composition status. In addition, the erythrocyte and breast milk measurements and the dietary analysis based on the FFQ allowed us to comprehensively determine the FA status.

\section{Conclusions}

Maternal obesity is related to the Western diet, characterized by the higher content of energy, SFA, and sugars. These dietary changes increase adipose tissue mass, metabolic disturbances (such as insulin resistance, inflammation, and oxidative stress), and the alteration of PUFA synthesis. Our results show that dietary n-3 PUFA, DHA, and AA concentration in erythrocytes phospholipids and breast milk are reduced in women with obesity during the last part of pregnancy and the first six months of lactation. Dietary strategies are urgently needed to improve diet quality in pregnant and lactating women with obesity. These strategies should focus on incrementing n-3 PUFA status (especially DHA) from foods and supplements.

Author Contributions: Conceptualization R.V. and R.C.; methodology R.V., R.C., K.A.B., C.B.; software C.B. and R.V.; validation R.V. and R.C.; formal analysis R.C.; K.A.B. and C.B.; investigation R.C., K.A.B., C.B., J.S., C.P. and R.V.; resources R.V.; data curation C.B. and R.V.; writing-original draft preparation R.C. and R.V.; writing-review and editing R.C. and R.V.; project administration R.V.; funding acquisition R.V.; All authors have read and agreed to the published version of the manuscript.

Funding: This research received no external funding.

Institutional Review Board Statement: Institutional Review Board of the Faculty of Medicine, University of Chile (Protocol \#073-2011) and by the Ethics Committee of the Clinical Hospital, University of Chile (Protocol \#507/11).

Informed Consent Statement: Informed consent was obtained from all subjects involved in the study.

Data Availability Statement: All data used and/or analyzed during the study are available from the corresponding author on request.

Conflicts of Interest: The authors declare no conflict of interest.

\section{References}

1. Harding, J.E. The nutritional basis of the fetal origins of adult disease. Int. J. Epidemiol. 2001, 30, 15-23. [CrossRef] [PubMed]

2. Dodd, J.M.; Deussen, A.R.; Louise, J. Optimising gestational weight gain and improving maternal and infant health outcomes through antenatal dietary, lifestyle and physical activity advice: The OPTIMISE randomised controlled trial protocol. BMJ Open 2018, 8, e019583. [CrossRef] [PubMed]

3. Li, Y.; Liu, X.; Modi, N.; Uthaya, S. Impact of breast milk intake on body composition at term in very preterm babies: Secondary analysis of the Nutritional Evaluation and Optimisation in Neonates randomised controlled trial. Arch. Dis. Child Fetal Neonatal Ed. 2019, 104, F306-F312. [CrossRef] [PubMed]

4. Rees, W.D. Interactions between nutrients in the maternal diet and the implications for the long-term health of the offspring. Proc. Nutr. Soc. 2019, 78, 88-96. [CrossRef] [PubMed]

5. Catalano, P.M.; Ehrenberg, H.M. The short- and long-term implications of maternal obesity on the mother and her offspring. BJOG 2006, 113, 1126-1133. [CrossRef]

6. Catalano, P.M.; Shankar, K. Obesity and pregnancy: Mechanisms of short term and long- term adverse consequences for mother and child. BMJ 2017, 356, j1. [CrossRef] [PubMed]

7. World Health Organization. Obesity and Overweight. Available online: https://www.who.int/news-room/fact-sheets/detail/ obesity-and-overweight (accessed on 15 September 2021).

8. Chen, C.; Xu, X.; Yan, Y. Estimated global overweight and obesity burden in pregnant women based on panel data model. PLoS ONE 2018, 13, e0202183. [CrossRef] [PubMed]

9. Kominiarek, M.A.; Peaceman, A.M. Gestational weight gain. Am. J. Obstet. Gynecol. 2017, 217, 642-651. [CrossRef]

10. Heslehurst, N.; Rankin, J.; Wilkinson, J.R.; Summerbell, C.D. A nationally representative study of maternal obesity in England, UK: Trends in incidence and demographic inequalities in 619,323 births, 1989-2007. Int. J. Obes. 2010, 34, 420-428. [CrossRef]

11. Catalano, P.M.; Farrell, K.; Thomas, A.; Huston-Presley, L.; Mencin, P.; de Mouzon, S.H.; Amini, S.B. Perinatal risk factors for childhood obesity and metabolic dysregulation. Am. J. Clin. Nutr. 2009, 90, 1303-1313. [CrossRef] [PubMed]

12. Lahti-Pulkkinen, M.; Bhattacharya, S.; Wild, S.H.; Lindsay, R.S.; Räikkönen, K.; Norman, J.E.; Bhattacharya, S.; Reynolds, R.M. Consequences of being overweight or obese during pregnancy on diabetes in the offspring: A record linkage study in Aberdeen, Scotland. Diabetologia 2019, 62, 1412-1419. [CrossRef] [PubMed] 
13. Goldstein, R.F.; Abell, S.K.; Ranasinha, S.; Misso, M.; Boyle, J.A.; Black, M.H.; Li, N.; Hu, G.; Corrado, F.; Rode, L.; et al. Association of gestational weight gain with maternal and Infant outcomes: A systematic review and meta-analysis. JAMA 2017, 317, 2207-2225. [CrossRef]

14. Dude, A.M.; Grobman, W.; Haas, D.; Mercer, B.M.; Parry, S.; Silver, R.M.; Wapner, R.; Wing, D.; Saade, G.; Reddy, U.; et al. Gestational weight gain and pregnancy outcomes among nulliparous women. Am. J. Perinatol. 2021, 38, 182-190. [CrossRef] [PubMed]

15. Flegal, K.M.; Carroll, M.D.; Kit, B.K.; Ogden, C.L. Prevalence of obesity and trends in the distribution of body mass index among US adults, 1999-2010. JAMA 2012, 307, 491-497. [CrossRef] [PubMed]

16. Riley, L.; Wertz, M.; McDowell, I. Obesity in Pregnancy: Risks and Management. Am. Fam. Physician 2018, 97, 559-561.

17. Catalano, P.M.; Presley, L.; Minium, J.; Hauguel-de Mouzon, S. Fetuses of obese mothers develop insulin resistance in utero. Diabetes Care 2009, 32, 1076-1080. [CrossRef] [PubMed]

18. Paredes, C.; Hsu, R.C.; Tong, A.; Johnson, J.R. Obesity and Pregnancy. Neoreviews 2021, 22, e78-e87. [CrossRef] [PubMed]

19. Chandrasekaran, S.; Neal-Perry, G. Long-term consequences of obesity on female fertility and the health of the offspring. Curr. Opin. Obstet. Gynecol. 2017, 29, 180-187. [CrossRef] [PubMed]

20. Catalano, P.M. Obesity, insulin resistance, and pregnancy outcome. Reproduction 2010, 140, 365-371. [CrossRef] [PubMed]

21. Fu, Y.; Liu, X.; Zhou, B.; Jiang, A.C.; Chai, L. An updated review of worldwide levels of docosahexaenoic and arachidonic acid in human breast milk by region. Public Health Nutr. 2016, 19, 2675-2687. [CrossRef] [PubMed]

22. Das, U.N. Influence of polyunsaturated fatty acids and their metabolites on stem cell biology. Nutrition 2011, 27, 21-22. [CrossRef] [PubMed]

23. Hadley, K.B.; Ryan, A.S.; Forsyth, S.; Gautier, S.; Salem, N., Jr. The Essentiality of Arachidonic Acid in Infant Development. Nutrients 2016, 8, 216. [CrossRef]

24. Echeverría, F.; Valenzuela, R.; Hernandez-Rodas, M.C.; Valenzuela, A. Docosahexaenoic acid (DHA), a fundamental fatty acid for the brain: New dietary sources. Prostaglandins Leukot. Essent. Fatty Acids. 2017, 124, 1-10. [CrossRef] [PubMed]

25. Sambra, V.; Echeverria, F.; Valenzuela, A.; Chouinard-Watkins, R.; Valenzuela, R. Docosahexaenoic and Arachidonic Acids as Neuroprotective Nutrients throughout the Life Cycle. Nutrients 2021, 13, 986. [CrossRef]

26. Nakamura, M.T.; Yudell, B.E.; Loor, J.J. Regulation of energy metabolism by long-chain fatty acids. Prog. Lipid Res. 2014, 53, 124-144. [CrossRef] [PubMed]

27. Demmelmair, H.; Koletzko, B. Importance of fatty acids in the perinatal period. World Rev. Nutr. Diet. 2015, 112, 31-47. [PubMed]

28. Araya, J.; Rodrigo, R.; Pettinelli, P.; Araya, A.V.; Poniachik, J.; Videla, L.A. Decreased liver fatty acid delta-6 and delta-5 desaturase activity in obese patients. Obesity 2010, 18, 1460-1463. [CrossRef] [PubMed]

29. Elizondo, A.; Araya, J.; Rodrigo, R.; Signorini, C.; Sgherri, C.; Comporti, M.; Poniachik, J.; Videla, L.A. Effects of weight loss on liver and erythrocyte polyunsaturated fatty acid pattern and oxidative stress status in obese patients with non-alcoholic fatty liver disease. Biol. Res. 2008, 41, 59-68. [CrossRef] [PubMed]

30. Gázquez, A.; Prieto-Sánchez, M.T.; Blanco-Carnero, J.E.; Ruíz-Palacios, M.; Nieto, A.; van Harskamp, D.; Oosterink, J.E.; Schierbeek, H.; van Goudoever, J.B.; Demmelmair, H.; et al. Altered materno-fetal transfer of 13C-polyunsaturated fatty acids in obese pregnant women. Clin. Nutr. 2019, 39, 1101-1107. [CrossRef] [PubMed]

31. Barrera, C.; Valenzuela, R.; Chamorro, R.; Bascuñán, K.; Sandoval, J.; Sabag, N.; Valenzuela, F.; Valencia, M.P.; Puigrredon, C.; Valenzuela, A. The Impact of Maternal Diet during Pregnancy and Lactation on the Fatty Acid Composition of Erythrocytes and Breast Milk of Chilean Women. Nutrients 2018, 10, 839. [CrossRef] [PubMed]

32. Atalah, E.; Castillo, C.; Castro, R.; Aldea, A. Proposal of a new standard for the nutritional assessment of pregnant women. Rev. Med. Chil. 1997, 125, 1429-1436. [PubMed]

33. WHO/FAO/UNU. Human Energy Requirements, Report of a Joint FAO/WHO/UNU Expert Consultation; WHO: Rome, Italy, 2014.

34. Food and Nutrition Board, Institute of Medicine. Dietary Reference Intakes: Guiding Principles for Nutrition Labeling and Fortification; Institute of Medicine of the National Academies: Washington, DC, USA, 2001; 224p.

35. Ministery of Health (MINSAL). Perinatal Guide, 1st ed.; MINSAL: Santiago, Chile, 2015; ISBN 978-956-348-076-4.

36. Cerda, R.; Barrera, C.; Arena, M.; Bascuñán, K.A.; Jimenez, G. Photographic Atlas of Chilean Food and Typical Preparations. In National Survey of Food Consumption, 1st ed.; Government of Chile, Ministry of Health: Santiago, Chile, 2010.

37. Bascuñán, K.A.; Valenzuela, R.; Chamorro, R.; Valencia, A.; Barrera, C.; Puigrredon, C.; Sandoval, J.; Valenzuela, A. Polyunsaturated fatty acid composition of maternal diet and erythrocyte phospholipid status in Chilean pregnant women. Nutrients 2014, 6, 4918-4934. [CrossRef] [PubMed]

38. Bligh, E.G.; Dyer, W.J. A rapid method of total lipid extraction and purification. Can. J. Biochem. Physiol. 1959, 37, 911-917. [CrossRef] [PubMed]

39. Ruiz-Gutierrez, V.; Cert, A.; Rios, J.J. Determination of phospholipid fatty acid and triacylglycerol composition of rat caecal mucosa. J. Chromatogr. 1992, 575, 1-6. [CrossRef]

40. Morrison, W.R.; Smith, L.M. Preparation of fatty acid methyl esters and dimethylacetals from lipids with boron fluorideMethanol. J. Lipid Res. 1964, 5, 600-608. [CrossRef]

41. Valenzuela, R.; Bascuñán, K.; Chamorro, R.; Barrera, C.; Sandoval, J.; Puigrredon, C.; Parraguez, G.; Orellana, P.; Gonzalez, V.; Valenzuela, A. Modification of docosahexaenoic acid composition of milk from nursing women who received alpha linolenic acid from chia oil during gestation and nursing. Nutrients 2015, 7, 6405-6424. [CrossRef] 
42. Chang, M.W.; Brown, R.; Nitzke, S. Fast Food Intake in Relation to Employment Status, Stress, Depression, and Dietary Behaviors in Low-Income Overweight and Obese Pregnant Women. Matern. Child Health J. 2016, 20, 1506-1517. [CrossRef]

43. Garmendia, M.L.; Alonso, F.T.; Kain, J.; Uauy, R.; Corvalan, C. Alarming weight gain in women of a post-transitional country. Public Health Nutr. 2014, 17, 667-673. [CrossRef]

44. Araya, M.B.; Padilla, O.; Garmendia, M.L.; Atalah, E.; Uauy, R. Prevalence of obesity among chilean women in childbearing ages. Rev. Med. Chil. 2014, 142, 1440-1448.

45. Godfrey, K.M.; Reynolds, R.M.; Prescott, S.L.; Nyirenda, M.; Jaddoe, V.W.; Eriksson, J.G.; Broekman, B.F. Influence of maternal obesity on the long-term health of offspring. Lancet Diabetes Endocrinol. 2017, 5, 53-64. [CrossRef]

46. Andres, A.; Hull, H.R.; Shankar, K.; Casey, P.H.; Cleves, M.A.; Badger, T.M. Longitudinal body composition of children born to mothers with normal weight, overweight, and obesity. Obesity 2015, 23, 1252-1258. [CrossRef] [PubMed]

47. Puentes, A.G.; Alemany, A.M.; Chisaguano, A.M.; Montes Goyanes, R.; Castellote, A.I.; Torres-Espínola, F.J.; García-Valdés, L.; Escudero-Marín, M.; Segura, M.T.; Campoy, C.; et al. The effect of maternal obesity on breast milk fatty acids and its association with infant growth and cognition-The PREOBE Follow-Up. Nutrients 2019, 11, 2154. [CrossRef] [PubMed]

48. Álvarez, D.; Muñoz, Y.; Ortiz, M.; Maliqueo, M.; Chouinard-Watkins, R.; Valenzuela, R. Impact of maternal obesity on the metabolism and bioavailability of polyunsaturated fatty acids during pregnancy and breastfeeding. Nutrients 2020, 13, 19. [CrossRef] [PubMed]

49. Valenzuela, R.; Videla, L.A. The importance of the long-chain polyunsaturated fatty acid n-6/n-3 ratio in development of non-alcoholic fatty liver associated with obesity. Food Funct. 2011, 2, 644-648. [CrossRef]

50. Gonzalez-Soto, M.; Mutch, D.M. Diet Regulation of Long-Chain PUFA Synthesis: Role of Macronutrients, Micronutrients, and Polyphenols on delta-5/delta-6 Desaturases and Elongases 2/5. Adv. Nutr. 2021, 12, 980-994. [CrossRef]

51. Gura, T. Nature's first functional food. Science 2014, 345, 747-749. [CrossRef]

52. Walker, A. Breast milk as the gold standard for protective nutrients. J. Pediatr. 2010, 156, S3-S7. [CrossRef]

53. Sheila, M.I. Human milk: Maternal dietary lipids and infant development. Proc. Nutr. Soc. 2007, 66, 397-404.

54. Koletzko, B.; Thiel, I.; Abiodun, P.O. The fatty acid composition of human milk in Europe and Africa. J. Pediatr. 1992, 120, S62-S70. [CrossRef]

55. Antonakou, A.; Skenderi, K.P.; Chiou, A.; Anastasiou, C.A.; Bakoula, C.; Matalas, A.L. Breast milk fat concentration and fatty acid pattern during the first six months in exclusively breastfeeding Greek women. Eur. J. Nutr. 2013, 52, 963-973. [CrossRef]

56. Campoy, C.; Chisaguano Tonato, A.M.; de la Garza Puentes, A.; Sáenz de Pipaón, M.; Verduci, E.; Koletzko, B.; González Casanova, I.; Larqué, E.; Valenzuela, R.; Moreno Villares, J.M.; et al. Controversy about the critical role of long-chain polyunsaturated fatty acids, arachidonic acid (ARA) and docosahexaenoic acid (DHA), during infancy. Nutr. Hosp. 2021, 38, 1101-1112. [PubMed]

57. Spector, A.A.; Kim, H.Y. Discovery of essential fatty acids. J. Lipid Res. 2015, 56, 11-21. [CrossRef] [PubMed]

58. Rincón-Cervera, M.Á.; Valenzuela, R.; Hernandez-Rodas, M.C.; Barrera, C.; Espinosa, A.; Marambio, M.; Valenzuela, A. Vegetable oils rich in alpha linolenic acid increment hepatic n-3 LCPUFA, modulating the fatty acid metabolism and antioxidant response in rats. Prostaglandins Leukot. Essent. Fatty Acids 2016, 111, 25-35. [CrossRef] [PubMed]

59. Bazinet, R.P.; Layé, S. Polyunsaturated fatty acids and their metabolites in brain function and disease. Nat. Rev. Neurosci. 2014, 15, 771-785. [CrossRef] [PubMed]

60. Das, U.N. Essential fatty acids: Biochemistry, physiology and pathology. Biotechnol. J. 2006, 1, 420-439. [CrossRef] [PubMed]

61. Valenzuela, R.; Rincón-Cervera, M.Á.; Echeverría, F.; Barrera, C.; Espinosa, A.; Hernández-Rodas, M.C.; Ortiz, M.; Valenzuela, A.; Videla, L.A. Iron-induced pro-oxidant and pro-lipogenic responses in relation to impaired synthesis and accretion of long-chain polyunsaturated fatty acids in rat hepatic and extrahepatic tissues. Nutrition 2018, 45, 49-58. [CrossRef]

62. Brei, C.; Stecher, L.; Much, D.; Karla, M.T.; Amann-Gassner, U.; Shen, J.; Ganter, C.; Karampinos, D.C.; Brunner, S.; Hauner, H. Reduction of the n-6:n-3 long-chain PUFA ratio during pregnancy and lactation on offspring body composition: Follow-up results from a randomized controlled trial up to 5 y of age. Am. J. Clin. Nutr. 2016, 103, 1472-1481. [CrossRef]

63. Hauner, H.; Much, D.; Vollhardt, C.; Brunner, S.; Schmid, D.; Sedlmeier, E.M.; Heimberg, E.; Schuster, T.; Zimmermann, A.; Schneider, K.T.M.; et al. Effect of reducing the n-6:n-3 long-chain PUFA ratio during pregnancy and lactation on infant adipose tissue growth within the first year of life: An open-label randomized controlled trial. Am. J. Clin. Nutr. 2012, 95, 383-394. [CrossRef]

64. Rahmawaty, S.; Lyons-Wall, P.; Charlton, K.; Batterham, M.; Meyer, B.J. Effect of replacing bread, egg, milk, and yogurt with equivalent $\omega-3$ enriched foods on $\omega-3$ LCPUFA intake of Australian children. Nutrition 2014, 30, 1337-1343. [CrossRef]

65. Miles, E.A.; Noakes, P.S.; Kremmyda, L.S.; Vlachava, M.; Diaper, N.D.; Rosenlund, G.; Urwin, H.; Yaqoob, P.; Rossary, A.; Farges, M.C.; et al. The salmon in pregnancy study: Study design, subject characteristics, maternal fish and marine n-3 fatty acid intake, and marine n-3 fatty acid status in maternal and umbilical cord blood. Am. J. Clin. Nutr. 2011, 94, 1986S-1992S. [CrossRef]

66. Sherry, C.L.; Oliver, J.S.; Marriage, B.J. Docosahexaenoic acid supplementation in lactating women increases breast milk and plasma docosahexaenoic acid concentrations and alters infant omega 6:3 fatty acid ratio. Prostaglandins Leukot. Essent. Fatty Acids 2015, 95, 63-69. [CrossRef] [PubMed]

67. Smit, E.N.; Koopmann, M.; Boersma, E.R.; Muskiet, F.A. Effect of supplementation of arachidonic acid (AA) or a combination of AA plus docosahexaenoic acid on breastmilk fatty acid composition. Prostaglandins Leukot. Essent. Fatty Acids 2000, 62, 335-340. [CrossRef] [PubMed] 
68. Monthé-Drèze, C.; Penfield-Cyr, A.; Smid, M.C.; Sen, S. Maternal pre-pregnancy obesity attenuates response to omega-3 fatty acids supplementation during pregnancy. Nutrients 2018, 10, 1908. [CrossRef] [PubMed]

69. Archer, E.; Marlow, M.L.; Lavie, C.J. Controversy and debate: Memory-Based Methods Paper 1: The fatal flaws of food frequency questionnaires and other memory-based dietary assessment methods. J. Clin. Epidemiol. 2018, 104, 113-124. [CrossRef] [PubMed]

70. Primorac, L.; Mandić, M.L.; Klapec, T.; Folivarski, K.; Perl, A.; Sudar, A. Adequacy of a food composition database to estimate fat and fatty acid intake. Int. J. Food Sci. Nutr. 2000, 51, 25-32. [CrossRef] [PubMed]

71. Pennington, J.A.T. Applications of food composition data: Data sources and considerations for use. J. Food Comp. Anal. 2008, 21, S3-S12. [CrossRef] 